\title{
$530^{\prime}$ \\ Recent Events in Guidance, Navigation and Control \\ by
}

Michael E. Polites

\begin{abstract}
This article summarizes recent events in Guidance, Navigation and Control (GN\&C) in space, weapons and missiles, and aircraft. The section on space includes recent developments with the following NASA spacecraft and space vehicles: Near Earth Asteroid Rendezvous, Deep Space 1, Microwave Anisotropy Probe, Earth Observer-1, Compton Gamma Ray Observatory, the International Space Station, X-38, and X-40A. The section on weapons and missiles includes recent developments with the following missiles: Joint Air-to-Surface Standoff Missile, Storm Shadow/Scalp EG precision standoff missile, Hellfire missile, AIM-120C Advanced medium-range air-to-air missile, Derby missile, Arrow 2, and the Standard Missile SM-3. The section on aircraft includes recent developments with the following aircraft: Joint Strike Fighter, X-31, V-22, Couger/Super Puma Mk. 2, Predator B 001, and the Unmanned Combat Air Vehicle.

The complete article is about 4000 words long. Information in it was gathered almost entirely from Aviation Week and Space Technology and Space News, which is already part of the public domain. A few paragraphs were submitted by NASA-Goddard, but they were cleared for public release. The article will appear in the proceedings of the 2001 Guidance, Navigation and Control Conference that is sponsored by the American Institute of Aeronautics and Astronautics.
\end{abstract}




\title{
Recent Events in Guidance, Navigation and Control by Michael Polites
}

\author{
In Space
}

Previously, the Near Earth Asteroid Rendezvous (NEAR) spacecraft, a joint venture of NASA and the Applied Physics Laboratory of Johns Hopkins University, became the first spacecraft to ever orbit an asteroid, in this case the asteroid Eros. More recently, it became the first spacecraft to ever land on an asteroid. To recount the events, it took NEAR about four years to travel a 2 billion-mile, looping route to reach and rendezvous with Eros. It then began orbiting the asteroid, a first of its kind in space, and orbited the 33x13x13-kilometers asteroid for a year, taking some 160,000 images and beaming them back to Earth. At the end of its expected life, scientists decided a landing attempt could provide some bonus science, although the spacecraft was never designed to land on the asteroid. Five thruster braking burns were made to reduce the speed of NEAR to a range of 2 to 7 miles per hour for descent. However, the asteroid rotates faster than this and has boulders, hills, and valleys on the surface. So, it was possible that the asteroid could slam into the spacecraft before it completed descent. This did not happen, the spacecraft landed safely, and continued to send signals back to Earth. Prior to these events, scientists had placed the chances that these events would be successful at about one in a hundred.

Jet Propulsion Laboratory's Deep Space 1 (DS1) spacecraft doubled its own record for uninterrupted rocket thrusting by running for 29 days and 3.7 hours its Hughes ion engine, which is boosting the spacecraft trajectory to fly by the comet Borrelly on September 22, 2001. The spacecraft normally fires the engine for a week and then pauses while turning itself for Earth communications. However, communications with the Earth were degraded for about a month due to superior conjunction with the Sun and so the engine ran nonstop without Earth turns. Because the onboard star tracker had failed, the main science camera, the Miniature Integrated Camera Spectrometer, was used for attitude determination and successfully held lock on a single star during this 29-day period.

During the summer of 2001, NASA-Goddard and Princeton University are scheduled to launch their Microwave Anisotropy Probe (MAP) spacecraft into a Lissajous orbit about the L2 Sun-Earth Lagrange point, about 1.5 million kilometers from Earth in the anti-Sun direction. This orbit was selected to minimize environmental disturbances, allow the microwave receivers to be passively cooled, and maximize observing efficiency. A Delta II launch vehicle will place the spacecraft into a 185-kilometer circular parking orbit with a 28.7-degree inclination. Transfer to the mission orbit will be accomplished with a series of phasing loops and a lunar gravity assist. Several new tools were developed to aid in designing trajectory maneuvers required during the phasing loops and in the mission orbit. A Monte Carlo analysis of the trajectory that allows placement of maneuvers at various combinations of phasing orbit perigees was developed and executed in order to guarantee the availability of trajectories that satisfy all mission requirements for all dispersions and within the fuel budget. To the knowledge of NASA-Goddard and Princeton, no one in the industry has ever performed a Monte Carlo analysis using flexible placement of maneuvers as solutions to the statistical problem.

NASA's first-ever autonomous formation flying mission is now under way with the launch of the Earth Observer-1 (EO-1) satellite. EO-1 was launched from Vandenberg Air Force Base (AFB) as a technology mission to fly in formation with Landsat-7 while also demonstrating technologies for electric propulsion (i.e. pulsed-plasma thrusters), onboard processing, and data storage. Both EO-1 and Landsat-7 carry instruments that enable scientists to study high-resolution images and climatic trends in the Earth's environment. The EO- 1 satellite flies only 60 seconds or 450 kilometers behind Landsat- 7 and maintains the separation within 2 seconds. This separation is necessary for EO- 1 to observe the same ground location through the same atmospheric region. Onboard EO-1 is an advanced software controller that is capable of autonomously planning, executing, and calibrating satellite orbit maneuvers to maintain the separation between the two satellites. This flight software provides for the ingest of real-time navigation data from the 
onboard Global Positioning System (GPS), the transfer of data from the maneuver algorithm for maneuver commands, onboard predictions of where the satellites will be in the future, and actual onboard commanding of the thruster firings.

NASA-Goddard is developing MEMS (Micro-Electro-Mechanical Systems) catalytic monopropellant propulsion systems to enable distributed Earth science and space science missions. These missions require micro-propulsive maneuvers for formation flying and precision pointing and the use of micro-, nano-, or pico-sized satellites. Current propulsion technology cannot meet the minimum thrust requirements (10-1000 milli-Newtons), the minimum impulse-bit requirements (1-1000 milli-Newton-seconds), the extremely limited system mass ( $<0.1$ kilograms), the extremely limited system volume ( $<1$ cubic-centimeters), and the power constraints $(<1$ watt) for these missions. When compared to other proposed micro-propulsion concepts, MEMS catalytic monopropellant thrusters show the promise of the combined advantages of high specific density, low system power and volume, large range of thrust levels, repeatable thrust vectors, and simplicity of integration and control. The current effort has been to utilize hydrogen peroxide as a propellant in a MEMS fabricated thruster. The targeted thrust level range is between 10 and 500 milliNewtons with impulse bits between 1 and 1000 micro-Newton-seconds and an $I_{\text {sp }}$ of greater than 110 seconds.

The NASA-Goddard and TRW Compton Gamma Ray Observatory (CGRO) successfully entered the Earth's atmosphere over the targeted Pacific Ocean. This is the first time NASA has conducted a controlled de-orbit of an unmanned spacecraft from low earth orbit. De-orbit was necessitated by the loss of one of the CGRO's three gyros and the fact that loss of a second gyro would have resulted in degraded attitude controllability. The criticality of this operation was heightened by the 14,000-kilogram mass of the spacecraft and the loss of two of the four orbit control thrusters soon after launch in 1991. The re-entry maneuver scenario consisted of engineering burns to verify thruster performance, followed by four $26+$ minute re-entry burns centered at apogee. The four re-entry burns successively reduced the perigee altitude to $350,250,150$, and 50 kilometers, respectively. CGRO completed its re-entry into the Pacific Ocean within 30 minutes after the fourth burn.

Software developed to reorient satellites whose gyroscopes had failed is being made available to extend the life and potentially reduce the cost of spacecraft. The Aerospace Corporation developed and patented a software package called Pseudogyro that emulates the function of hardware gyros. It was originally developed for a U.S. government national security satellite. Prelaunch analyses predicted the satellite's gyros would fail sooner than expected. So, Pseudogyro was installed as a supplemental system, allowing the operators to turn off the gyros periodically in order to extend their life. This saved the U.S. government about $\$ 15$ million, the cost incurred if the gyros had been replaced before launch. Pseudogyro was then used to extend the life of the U.S. National Reconnaissance Office's Space Technology Experiment satellite after the satellite's primary and secondary gyroscopes failed. ESA has a patent pending for a similar software package developed by Astrium SAS of Velizy, France for the European-U.S. Solar and Heliospheric Observatory (Soho) satellite after its gyros began failing.

The Charles Stark Draper Laboratory (CSDL) developed software algorithms that allowed the Space Shuttle to successfully boost the orbit of the International Space Station (ISS). The algorithms were designed to keep the Shuttle pulse firings short in order to avoid damaging the ISS solar arrays. The Space Shuttle can provide the orbit boost capability by using extra propellant that it routinely carries for contingencies. Ground crews determine the altitude that the ISS should be boosted each time. CSDL determined that this technique could be used for throughout the ISS assembly process for maintaining orbit.

The ISS assembly process will be aided by the Canadian Space Agency's (CSA's) new Space Station Remote Manipulator System (SSRMS), which was recently delivered to the ISS by the Space Shuttle and its crew. The SSRMS is a bigger, better, and smarter version of the Space Shuttle Remote Manipulator System (RMS), which was also developed by the CSA. The SSRMS is 8 feet longer than the 50-foot RMS and has force-moment-sensing software so its operators can tell how much force it is exerting. Unlike the RMS, the ISS crew operating the SSRMS will not be able to see it directly. Instead, they must rely totally on television, computer graphics, and digital readouts to operate it. Unlike the fixed RMS, the SSRMS has 
mechanical end-effectors on both ends that allow it to walk like an inchworm around the exterior of the ISS. Later, it will be able to ride on its rail-mounted base system.

NASA-Johnson's X-38, an 80\%-scale model of the Crew Return Vehicle (CRV) that is to provide emergency escape for International Space Station (ISS) crews, had its first free flight. The vehicle was released from a B-52 aircraft, automatically stabilized under the preprogrammed deployment of a drogue chute, and successfully landed under parafoil control on a dry lakebed at Edwards AFB. After release, the vehicle did experience an unplanned 360-degree roll at a roll rate of about 20 degrees/second. Actual separation from the B-52 was clean and the flight control system did maintain Angle Of Attack (AOA) throughout the roll. A software problem in the flight control system was suspected, but aerodynamic disturbances immediately after separation could have played a role in the anomaly.

NASA and The Boeing Company had seven successful drop tests of the X-40A, a test vehicle built by Boeing for the U.S. Air Force (USAF). The X-40A was originally developed under the USAF Space Maneuver Vehicle Technology Program and has been on loan to NASA for drop tests intended to mitigate flight testing the X-37, a robotic space plane that will be capable of reentering the Earth's atmosphere from orbit and landing autonomously. The X-37 will be built by Boeing under NASA-Marshall management and is intended to demonstrate technologies for future-generation reusable launch vehicles that could reduce the cost of access to space. In the drop tests, conducted at NASA-Dryden and Edwards AFB, the engineless X40A was carried to an altitude of 15,000 feet by an Army CH-47D Chinook helicopter and released. It descended and landed autonomously on a runway at Edwards. The tests validated simulation models extremely well and validated several technologies planned for the X-37. These include the Computed Air Data System, a high fidelity air data system to be used during orbital reentry, and the Honeywell Space Integrated GPS/Inertial Navigation System (INS), or SIGI, used for navigation.

A NASA-Goddard Wallops Flight Facility (WFF) payload was launched from Esrange, near Kiruna, Sweden, on an Improved Orion sounding rocket. The mission had three purposes: to demonstrate the ability of the WFF inverse Differential-GPS (D-GPS) system to replace radar systems at Esrange for metric tracking, telemetry antenna pointing and impact prediction; to flight test a new low cost Commercial-OffThe-Shelf (COTS) GPS receiver; and to demonstrate space-based vehicle tracking using the WFF Flight Modem. The mission was successful in all these aspects. Range safety personnel were able to process the data and used it as the prime tracking data source. The COTS receiver, while not as adept to high dynamic tracking as the standard system flown, shows potential for further development. The Flight Modem was the first use of a commercial communications satellite for tracking a sounding rocket payload. Although the commercial operator's local ground station is not yet enabled to pass digital data, GPS data was transmitted to the satellite error free. An additional GPS receiver built by DLR of Germany was flown on the mission, giving an unprecedented opportunity to compare results from three GPS receivers on the same rocket. Papers on the GPS and the Flight Modem are to be presented at conferences in Laurel, Maryland; Albuquerque, New Mexico; and Biarritz, France.

\section{In Weapons and Missiles}

The USAF's new Joint Air-to-Surface Standoff Missile successfully completed its first test, using its imaging InfraRed (IR) terminal seeker to locate its target. The missile was launched from an F-16D aircraft while flying at an altitude of 15,000 feet and a speed of Mach 0.8. The missile was GPS guided through three navigation way points before diving at a 70-degree angle.

The Storm Shadow/Scalp EG precision standoff missile successfully completed its first test firing, moving Europe closer to obtaining its own cruise missile capability. The missile was fired from a Mirage $2000 \mathrm{~N}$ aircraft flying at 20,000 feet and Mach 0.9 and hit its target more than 135 miles away.

The USAF successfully fired Army Hellfire anti-armor missiles from the Predator Unmanned Air Vehicle (UAV), developed by the U.S. Navy (USN). The Predator fired a Hellfire missile for the first time in flight at an altitude of 2,000 feet and a speed of 70 knots and hit a tank that was 3 miles away. The tank was 
designated by a ground-mounted laser. In second and third firings, which were also successful, the tanks were designated by a laser mounted on the Predator. Plans are to raise the launch altitude up to 15,000 feet, which is more likely for combat since a number of Predators were shot down in Serbia when the aircraft had to drop under low ceilings to make observations.

The USAF completed the first test launch of the AIM-120C Advanced medium-range air-to-air missile (Amraam). The unguided missile was fired from an F-22 flying wings level at an altitude of 15,500 feet and a speed of Mach 0.9. The test was considered significant because it confirmed the reconfigured AIM-120C could be fired successfully from tight quarters in the main weapons bay of the F-22. The C-model was developed specifically for internal carriage on the F-22 and features clipped wings and fins.

Israel's Rafael unveiled its Derby beyond-visual-range air-to-air missile that employs an active radar seeker developed by the Israel Aircraft Industries. The missile can be operated in two modes. One is the lock-on after launch mode for long-range engagements in which the missile employs inertial guidance immediately after launch until the seeker is activated and homes in on the target. The other is the lock-on before launch mode for short engagements. Here the seeker is activated before launch and guides the missile all the way to the target. The seeker can be slaved to the aircraft's radar or the pilot's helmet-mounted cueing system. Derby has undergone flight testing on an Israel Air Force F-16 and is adaptable to other lighter-weight fighters such as the F-5 and the Mirage.

Israel's Arrow 2 anti-tactical ballistic missile interceptor successfully intercepted a ballistic missile target. This was the second successful intercept for the Arrow 2 and the first against the new, air-launched, inbound Black Sparrow target, which was launched from an Israel Air Force F-15 over the Mediterranean Sea. This was one of the most realistic and operationally oriented tests to date, in that it was the first time this weapon was used to intercept an incoming target that was headed for Israel.

The USN and its prime contractor Raytheon had a successful flight test of the Standard Missile SM-3 ballistic missile interceptor. The test was designed strictly to evaluate the stability and control of the SM-3 airframe through kill-vehicle separation. There was a target, but no intercept was attempted and the kinetic kill vehicle that should intercept the target did not carry a real divert and attitude control system. Nevertheless, the USS lake Erie cruiser that launched the SM-3 was able to guide it to less than 400 meters from the target, a range smaller than what is required. The test began with the launch of the Orbital Sciences' Aries target at the Navy's Pacific Missile Range Facility near Kauai, Hawaii. The USS lake Erie was 200 nautical miles down range at the time. The Aegis SPY-1B radar, built by Lockheed Martin, detected the target and six minutes later, the SM-3 was launched. At an altitude of 90 kilometers, the nose cone separated, exposing the long-wave IR seeker, which is used to guide the kill vehicle to its target during the final seconds of flight. After about 500 seconds, the kill vehicle separated from the third stage. Performance of the entire system appeared to be nominal.

Lockheed Martin Space Systems in Sunnyvale, CA opened a 16,000 square feet test facility for the beam control and fire control systems that it is providing for the USAF AirBorne Laser (ABL) theatre ballistic missile defense system. The high-energy laser beam will be fired through a turret mounted in the nose of a Boeing 747-400 Freighter and the new test facility will be used to thoroughly wring out the beam control and fire control systems end-to-end prior to their integration into the aircraft. The first chance to actually demonstrate the ABL concept against a flying target is expected in late 2003 with the aircraft flying out of Edwards AFB and the target launched from Vandenberg AFB, heading along the southern California coast.

\section{In Aircraft}

The Boeing and Lockheed Martin Joint Strike Fighter (JSF) flight demonstration programs are fully underway. Each company has two prototype aircraft, but is using them in different ways. Boeing built an X32A to show Conventional TakeOff and Landing (CTOL) capabilities for the USAF and carrier approach flying qualities for the USN. They also built an X-32B to demonstrate Short TakeOff and Vertical Landing (STOVL) capabilities, which is achieved by closing the rear exhaust nozzle and redirecting thrust 
downward through two lift nozzles. Lockheed Martin, on the other hand, built an X-35A aircraft to demonstrate CTOL capabilities for the USAF and an X-35C, with a bigger wing and larger control surfaces than the X-35A, to demonstrate carrier approach and landings for the USN. Lockheed also converted its X$32 \mathrm{~A}$ to an X-32-B STOVL demonstrator by adding a lift fan and a flexible tail nozzle, among other things.

The U.S.-German X-31 aircraft returned to flight after a six-year hiatus to demonstrate Extremely Short Take Off and Landing (ESTOL) capability. The X-31 is used because of its thrust vectoring, which is critical for demonstrating ESTOL. It can fly at AOAs up to 70 degrees and speeds up to Mach 1.3. Investigators are exploring ESTOL capabilities in order to increase military aircraft safety and operational flexibility and to cut life-cycle costs. Specifically, an ESTOL aircraft would be able to: land on carrier decks at speeds lower than current aircraft, thereby reducing aircraft wear and tear; reduce the need for wind-over-the-deck landing operations; eliminate or reduce the need for aircraft to dump stores before landing on a carrier; and lower carrier arresting-gear and catapult-maintenance requirements.

The crash of a V-22 tiltrotor aircraft has grounded the fleet that supports USAF and U.S. Marine operations. The aircraft was on its final approach into the Marine Corps Air Station New River, NC and experiencing a leak in its hydraulic system that drives flight-critical systems, such as actuators changing the angle of the nacelles and swashplate actuators, which are used to control collective and cyclic blade pitch that allow thrust control when in the airplane mode. At the moment the pilot started converting the aircraft from its forward-flight, fixed-wing mode into its helicopter mode for landing, the hydraulics failed. The pilot pushed the rotors from the $90 \%$ fixed-wing mode, that they had reached, back to full $100 \%$ fixed-wing settings, but the aircraft crashed moments later, nonetheless. As a result of an investigation into the crash, engineers were told to reexamine, improve, and debug the hydraulic systems and the flight control software on the aircraft.

Eurocopter began flight testing an improved Couger/Super Puma Mk. 2 helicopter that is intended to form the core of France's search-and-rescue helicopter fleet. This version offers the following improvements over the previous one: 900 pounds more internal payload, 22 knots additional speed, and 147 nautical miles additional range. Range can also be enhanced by inflight refueling, which has been successfully demonstrated. The engines of the improved Couger/Super Puma Mk. 2 feature duplex full-authority digital control and drive a new five-blade main rotor offering reduced vibration levels.

The USN is testing a shipboard-based GPS system that will give pilots precision, jam-free guidance for automatic carrier landings. The system being tested is the USN configuration of Raytheon's Joint Precision Approach \& Landing Systems (JPALS), modified to add relative D-GPS to compensate for ship motion. USN test pilots made the first automatic carrier landings with this system in an F/A-18 at Naval Air Station Patuxent River, MD. The chief test pilot for JPALS said that it matched or exceeded the performance of the current automatic carrier landing systems.

Researchers at the NASA-Ames neuroengineering laboratory successfully simulated the landing of a Boeing 757 aircraft using neuroelectric control. In the simulation, the pilot controlled the aircraft with muscle movements read by eight electrodes implanted in an armband on his forearm. The outputs from the electrodes were input into neural net software developed at Ames that allowed his hand and arm movements to control the aircraft as if he were grasping a joystick. The advantage of neuroelectric control is that human nerve signals can be linked directly with devices without the aid of joysticks or mice, thereby providing rapid, intuitive control.

NASA-Langley and Lockheed Martin flight demonstrated an advisory technology that could increase the safety of rollout and turnoff and hold short runway operations for all weather conditions. These tests were done at Dallas-Fort Worth with Langley's research Boeing 757 aircraft. On approach to landing, the Hold Short Advisory Landing Technology (HSALT) system, which utilizes D-GPS, computes and displays information on a head-up, as well as a head-down, display to help pilots decide if the plane can stop safely. After touchdown, deceleration and situational information help the pilot to stop the plane safely, before reaching the hold short, or to exit the runway earlier. 
Lockheed Martin completed flight testing the Advanced Fighter Technology Integration (AFTI) F-16 equipped with electrohydrostatic control surface actuators and an advanced electrical power generation system. According to Lockheed Martin, this is the first airplane to fly with "power-by-wire" hardware and software. If the Lockheed Martin JSF team wins the engineering and manufacturing development contract, they plan to install the actuators and power generation system in their JSF production airplanes.

The Predator B 001 drone made its first flight at General Atomics' desert runway near the El Mirage dry lakebed in Southern California. Except for some poor throttle linearity, the flight was flawless. The B 001 is the first in a series of three new UAVs for NASA's Environmental Aircraft and Sensor Technology Program. The aircraft is expected to be capable of carrying 750 pounds to an altitude of 50,000 feet.

The Department of Defense demonstrated for the first time a method for freeing the use of UAVs from their dedicated ground stations, which could spur the use of UAVs in combat. The demonstrations involved a Predator UAV controlled entirely by a generic ground control station, called a Tactical Control Station (TCS), in place of the Predator's dedicated ground station. At the U.S. Army's Fort Huachua, Arizona, nine Predator missions were flown. Of these, the TCS was used for nine takeoffs and eight landings. The ninth landing was accomplished using the Predator's dedicated ground station, after control of the UAV was handed off to it from the TCS. The TCS, developed by the USN, gives users control over a number of different UAVs and allows one type of UAV to replace another in an area of operations without having users on the ground or at sea change ground stations.

Northrup Grumman designed an Unmanned Combat Air Vehicle (UCAV) with an unorthodox kite or diamond shape and plans to build a flight demonstrator of it. Their goal is to demonstrate aerodynamic characteristics suitable for USN aircraft carrier takeoffs and landings. They believe this shaped vehicle is probably the most complex for flying off a carrier. Full-sized versions of the demonstrator could have wingspans of more than 60 feet. Top and bottom surfaces would have inlaid flaps forward of the elevons that act as a rudder and vertical stabilizer. The USN is interested in UCAVs launched from carriers for surveillance missions. 